\title{
Lung Transplantation as An Intervention for Pediatric Pulmonary Hypertension
}

\author{
Ernestina Melicoff ${ }^{1}$, Don Hayes ${ }^{2}$, and Christian Benden ${ }^{3}$ \\ ${ }^{1}$ Baylor College of Medicine \\ ${ }^{2}$ University of Cincinnati \\ ${ }^{3}$ University of Zurich
}

May 7, 2020

\begin{abstract}
Lung transplantation is recognized as a treatment option for selected patients with end-stage lung disease and pulmonary vascular disorders. Overall, pulmonary hypertension $(\mathrm{PH})$ is the second most frequent indication for children requiring lung transplantation. Pediatric lung transplantation can be complex; in PH patients timing for listing and the perioperative management can be particularly challenging making interdisciplinary collaboration with the referring PH team essential. Because pediatric patients typically have preserved cardiac index and exercise tolerance even with advanced disease, they should be referred early even if they do not meet the proposed criteria for listing by ISHLT published in 2015: NYHA functional class III or IV without improvement, cardiac index $<2$ liters $/ \mathrm{min} / \mathrm{m}^{\wedge} 2$, mean right atrial pressure of $>15 \mathrm{mmHg}$. Bridging strategies with extracorporeal support should be determined at time of listing prior to transplantation anticipating the possibility of clinical deterioration. Bilateral lung transplantation using cardiopulmonary bypass to provide hemodynamics stability is the standard practice in pediatric centers. The immediate post-transplant period is characterized by dramatic normalization of PVR as well as changes in the RV and LV physiology and function which can be life-threatening. Induction, immunosuppression, prophylaxis and surveillance are not different from patients without PH. Overall, outcomes in pediatric lung and heart-lung transplant patients for $\mathrm{PH}$ are not different from those children undergoing lung or heart-lung transplant for other indications. In fact, long-term survival is superior in children with idiopathic PH compared to other diseases, there is also a dramatic improvement in quality of life in most recipients
\end{abstract}

\section{INTRODUCTION}

Pediatric lung transplantation has been performed in selected centers for three decades, and it is today an accepted therapy in carefully selected pediatric candidates with progressive pulmonary and pulmonary vascular disease, delivering an overall survival benefit and improved health-related quality of life ${ }^{1-5}$. More than 100 lung transplant procedures are performed in the pediatric age group per year worldwide according to the most recent Thoracic Transplant Registry Report of the International Society for Heart and Lung Transplantation (ISHLT) ${ }^{6}$. Pediatric lung transplants are performed predominately at North American centers; in Europe, leading pediatric lung transplant programs are located in Hannover, London, and Vienna. However, the majority of centers in North America, Europe and Australia do five or fewer pediatric lung transplants per year and mostly candidates older than age five years ${ }^{6}$. Overall, cystic fibrosis $(\mathrm{CF})$ is the most common underlying disease in pediatric lung transplantation ${ }^{6}$. In children between one and 10 years of age, CF and idiopathic pulmonary arterial hypertension (IPAH) are the most common underlying primary indications for lung transplantation. Overall, IPAH is the second most frequent indication for children requiring lung transplantation, and in children aged one to five years IPAH and other forms of $\mathrm{PH}$ are the most common indication ${ }^{6}$. 
In general, pediatric lung transplant is an exceedingly complex medical-surgical endeavor requiring a multidisciplinary approach and close interdisciplinary collaboration. The surgical approach is often challenging due to previous surgery, the pre-transplant morbidities of the transplant candidates and the frequency of imperfect donor-recipient lung size matching. Additionally, overseeing and modulating immunosuppression in infants and children with developing immune systems with frequent viral infections is imposing. In addition, psychosocial aspects are potential hurdles to achieve good outcomes, particularly in adolescents ${ }^{1}$.

\section{CANDIDATE SELECTION AND LISTING}

Timing for referral and listing is always challenging for lung transplant candidates, particularly with any form of pulmonary hypertension (PH). $\mathrm{PH}$ as an indication for lung transplanation highlights the importance of interdisciplinary collaboration between the referring pulmonary hypertension and transplant center teams. As a general rule, patients should be considered for transplantation when they are at high risk of death within 1 to 2 years or have unacceptable quality of life, can survive the wait for organs and the surgery itself and have a high chance of living a near normal life after lung transplantation ${ }^{7}$. Patients with severe forms of PH like alveolar capillary dysplasia, pulmonary veno-occlusive disease (PVOD), pulmonary capillary hemangiomatosis and pulmonary vein stenosis, can progress rapidly to respiratory failure, making early referral vital ${ }^{8}$. Patients with IPAH or other forms of pulmonary vascular disease that meet the following criteria should be referred for evaluation: NYHA functional class III or IV, rapidly progressive disease, and optimized use of targeted pulmonary hypertension treatment (Table $\mathbf{1}$ ). Early referral gives the lung transplant e and families the opportunity to address barriers identified during evaluation. Contraindications to pediatric lung transplant include conditions that predispose the patient to a poor post-transplant outcome, such as poor adherence to therapy and concomitant organ failure not suitable to transplant ${ }^{8}$. Timing of listing should take into consideration waiting time (patient's blood type and size, local policy regarding priority ${ }^{9}$ ), center's expertise related to extracorporeal support ${ }^{10}$ and the patient's disease trajectory. Listing should be considered when patients meet the following criteria ${ }^{7}$ : NYHA functional class III or IV without improvement after 3 months of combination therapy including prostanoids, cardiac index $<2 \mathrm{liters} / \mathrm{min} / \mathrm{m}^{\wedge} 2$, mean right atrial pressure of $>15 \mathrm{mmHg}$. However, it has been noted that pediatric patients often have preserved cardiac index and no elevation of right atrial pressure even with advanced disease ${ }^{11}$. Furthermore, children often have preserved exercise tolerance at a later stage of disease than adults making exercise intolerance potentially too late a marker of severe disease ${ }^{12}$. Other risk factors that predict poor survival include: significant hemoptysis, pericardial effusion, progressive right heart failure, von Willebrand factor levels of $>240 \%$, elevated uric acid levels, and persistently elevated plasma levels of brain natriuretic peptide $>180 \mathrm{pg} / \mathrm{mL}^{8}$.

\section{WAIT LIST MANAGEMENT AND BRIDGING STRATEGIES}

Waitlist management is aimed at increasing the patient's prospect of survival while waiting and improving post-transplant outcomes. In general, it includes nutritional, physical rehabilitation and adjustments of targeted PH pharmacotherapy as indicated. For pulmonary vascular disorders the interventions are aimed at maximizing pulmonary blood flow and improving oxygen delivery and may include diuretics, anticoagulation, supplemental oxygen, and other therapies targeted at the pulmonary vasculature. Atrial septostomy and Pott's shunt procedures are reserved for patients with refractory pulmonary hypertension ${ }^{13}$, these therapies will be further discussed in other manuscripts in this Supplement \{references from the Editor\}.

Extracorporeal life support is an invasive, potentially life-sustaining technology that can be used in patients with persistent right ventricular failure without response to medical treatment. Historically veno-arterial extracorporeal membrane oxygenation (VA-ECMO) was considered a contraindication as a bridge to lung transplantation due to poor outcomes ${ }^{14}$. Survival has improved due to advances in ECMO technology, anticoagulation and enhanced experience in leading pediatric centers with high priority ECLS teams. Venovenous ECMO has been used in selected clinical PH patients whereby oxygenated blood can be directed through a large atrial septal defect into the left atrium. More recently the group at Texas Children's Hospital bridged two PVOD patients with VA-ECMO using central cannulation approach (Gazzaneo MC, personal communication). Preliminary discussion about bridging strategies should take place during the 
evaluation and should be determined by the time of listing in the event of clinical deterioration before organs become available. These bridging strategies should be limited to centers with standardized best practice protocols ${ }^{15}$.

\section{OPERATIVE STRATEGIES AND BLTX VS HLTX}

The standard surgical approach for patients with $\mathrm{PH}$ is bilateral lung transplantation under cardiopulmonary bypass. Heart-lung transplantation is usually only performed in patients with $\mathrm{PH}$ and irreparable cardiac defect ${ }^{16}$. Furthermore, heart-lung blocs have been increasingly rare in the USA with seven or fewer heartlung transplants performed in the pediatric age group in each of the last ten years (UNOS.org reference). Furthermore, clinical experience has shown good recovery of cardiac function in virtually all infants, children and adolescents with severe $\mathrm{PH}$ who survive the early post-operative period. Single lung transplant is rarely performed in pediatrics, particularly for patients with $\mathrm{PH}$ due to adverse outcomes like pulmonary edema and graft dysfunction ${ }^{17}$.

Cardiopulmonary bypass (CPB) is used in pediatric patients due to challenges utilizing double lumen endobronchial tubes in small airways to provide single lung ventilation ${ }^{17}$. As well, in PH patients, CPB provides hemodynamic stability in presence of increased pulmonary vascular resistance (PVR) ${ }^{18}$. In some adult transplant centers, ECMO has become the preferred intra-operative support as it is associated with reduced complications and improved survival ${ }^{19}$. ECMO can also be extended post-operatively to support patients either to prevent or in selected patients with significant graft dysfunction ${ }^{20}$.

\section{POST- (H)LTX MANAGEMENT}

Physiologic changes observed immediately after lung implantation with normalization of PVR should be anticipated; there is an increase in cardiac output and increase in left ventricular (LV) filling with acute LV dilation. This acute change in myocardial length-tension relationship can lead to LV failure. LV failure predisposes to elevation in post-capillary pulmonary vascular pressures which can lead to pulmonary edema and classic features of primary graft dysfunction. At the same time, RV dilation is immediately reduced resulting in a different length-tension alteration which may also compromise RV function, which is commonly compromised going into surgery. Treatment includes protective ventilator strategies, appropriate pressor support and fluid management to maintain normovolemia; beta-blockers may be considered to limit cardiac output. Post-operative ECMO support should be considered within 48 hours for patients with unrelenting low cardiac output and/or low lung compliance requiring $\mathrm{PIP}>35 \mathrm{cmH} 2 \mathrm{O}$ and $\mathrm{FiO} 2>0.6{ }^{15}$. Induction, immunosuppression, prophylaxis and surveillance are not different from patients without $\mathrm{PH}^{8}$.

\section{PATIENT OUTCOMES}

In 1992, the group at St. Louis Children's Hospital reported successful lung transplantation for advanced pulmonary hypertension in five children with congenital heart disease ${ }^{21}$. An early study published in 1996 examined outcomes in 24 children with pulmonary hypertension for lung transplantation and reported that seven patients did not meet criteria for listing with seven eventually transplanted ( 8 died, 2 still active on waitlist at time of study) ${ }^{22}$. Using both univariate and multivariate analysis (Cox regression), these investigators identified poor functional status at the time of referral defined as New York Heart Association (NYHA) Functional Class III or IV was significantly associated with and predicted death before transplant ${ }^{22}$. An important conclusion at that time when lung transplantation was in early development as a treatment option for children with PH, especially candidates with advanced pulmonary hypertension, was that late referral was a major risk factor for death on the waiting list and for early complications after implantation. In a subsequent study from St. Louis published in 2002 of 100 patients after lung transplantation for $\mathrm{PH}, 45$ patients with $\mathrm{PH}$ had higher early mortality post-transplant than other pulmonary indications for transplant ${ }^{23}$. A single-center study of 53 pediatric lung and heart-lung transplant recipients in Hannover, Germany found that IPAH as an indication for transplant did not significantly influence post-transplant mortality in both univariate and multivariate analyses ${ }^{24}$.

Investigating the effect of lung transplantation on IPAH, another report from St. Louis examined 26 children 
(1.6-18.9 years of age) referred for lung transplant with 19 transplanted and seven died on the waitlist $\mathrm{t}^{25}$. Median survival for IPAH compared favorably to all pediatric lung transplant recipients, 5.8 vs. 4.5 years ${ }^{25}$. The analysis by these investigators found that survival was independent of pre-transplant variables, which included age, weight, intravenous inotropes, intravenous pulmonary vasodilators, and right-sided cardiac pressure severity ${ }^{25}$. For those children who died on the waitlist, the differences compared to the transplanted group included significantly higher incidence of supra-systemic right heart pressures and hemoptysis ${ }^{25}$.

A multi-center, retrospective study by the International Pediatric Lung Transplant Collaborative evaluated outcomes of lung transplant for IPAH in 23 children IPAH with median survival to children who underwent heart-lung transplantation for pulmonary vascular disorders and lung transplantation for other indications ${ }^{26}$. These investigators reported an overall mortality of $4 \%$ at 3 months post-transplant with a median survival for children of 45 months $(2-123)^{26}$.

To summarize our review of the literature of studies investigating lung transplant outcomes for $\mathrm{PH}$ in children shows cohort size studies vary from five to 23 children, with one-year post-lung transplant survival rates ranging between $87-100 \%$. Schaellibaum et al ${ }^{26}$ estimated a 3 -year survival of $84 \%$, and Goldstein et $\mathrm{al}^{25}$ reported a 5 -year survival of $61 \%$. With limited literature on this important topic in the pediatric population, a recent study was performed using the United Network for Organ Sharing (UNOS) Registry to evaluate outcomes for children with IPAH after lung and heart-lung transplantation ${ }^{27}$. Between May 2005 and December 2015, 75 children (10 6 years) underwent transplant for IPAH (47 lung transplant, 18 heartlung transplant) with 5-year survival rates of $61 \%$ for lung transplant and $48 \%$ for heart-lung transplant, with no differences found in survival comparing lung to heart-lung transplant $(p=0.680)^{27}$.

Looking at the most recent report of the ISHLT Registry, children with IPAH had the best post-lung transplant survival as determined by the Kaplan-Meier method (Figure 1 ) compared to other pulmonary diagnoses ${ }^{28}$. However, the cohort of children with PH not IPAH had inferior outcomes across all indications for lung transplant (Figure 1 ) ${ }^{28}$. Limiting the analysis to conditional one-year post-lung transplant survival, children with IPAH had a median survival of 12.4 years with a signficant improvement in the outucomes for children with PH not IPAH (Figure 2 $)^{28}$.

The overall survival in the lung transplant and heart-lung transplant cohorts was not significantly different from survival among children undergoing lung transplant or heart-lung transplant for other indications, respectively ${ }^{27}$. Older age at transplant was associated with significantly lower mortality after transplantation $(p=0.036)$, while no clinical factors such as lung allocation score for children $>12$ years of age), status $1 \mathrm{~A}$ listing (heart-lung transplant cohort) and mean pulmonary artery pressure significantly influenced survival 27. In the 54 children who were listed during the study period who did not undergo transplant, 22 died, 14 experienced improvement of their condition, three refused transplant, and four deteriorated so that they were too sick to transplant ${ }^{27}$.

Investigating critically ill pediatric patients who underwent lung transplant, another St. Louis study examined outcomes of children regardless of underlying diagnosis requiring preoperative and post-operative ECMO ${ }^{14}$. The IPAH group was the largest who received preoperative ECMO and had the best survival rate after lung transplant ${ }^{14}$. IPAH trailed only cystic fibrosis as the largest group requiring post-operative ECMO in children who were critically ill after lung transplant, while remaining as the indication with the best survival rate ${ }^{14}$.

\section{Perspectives}

New advances in echocardiographic imaging techniques ${ }^{12}$ and in cardiac MRI ${ }^{29}$ may provide the tools to more accurately characterize patients with advancing disease to facilitate the difficult decision of the timing of referral of such patients to transplant centers.

Acknowledgements None.

Conflicts of interest 
The authors have no conflict of interest to declare.

\section{REFERENCES}

1. Benoit TM, Benden C. Pediatric lung transplantation: supply and demand. Curr Opin Organ Transplant 2019; 24: 324-328.

2. Mendeloff EN. The history of pediatric heart and lung transplantation. Pediatr Transplant 2002; 6: 270-279.

3. Benden C. Pediatric lung transplantation. J Thorac Dis 2017; 9: 2675-2683.

4. Hayes D Jr, Benden C, Sweet SC, et al. Current state of pediatric lung transplantation. Lung 2015; 193: 629-637.

5. Schmid FA, Benden C. Special considerations for the use of lung transplantation in pediatrics. Expert Rev Respir Med 2016; 10: 655-662.

6. Goldfarb SB, Hayes D Jr, Levvey BJ, et al. The International Thoracic Organ Transplant Registry of the International Society for Heart and Lung Transplantation: Twenty-first Pediatric Lung and Heart-Lung Transplantation Report - 2018; Focus Theme: Multiorgan Transplantation. J Heart Lung Transplant 2018; 37: 1196-206.

7. Weill D, Benden C, Corris PA, et al. A consensus document for the selection of lung transplant candidates:2014 - An update from the Pulmonary Transplantation Council of the International Society for Heart and Lung Transplantation. J Heart Lung Transplant 2015;34:1-15

8. Sweet SC. Pediatric Lung Transplantation. Respir Care. 2017 Jun;62(6):776-798

9. Egan TM, Murray S, Bustami RT, et al. Development of the new lung allocation system in the United States. Am J Transplant 2006; 6: 1212-1227.

10. Coleman R and Checchia P. ECMO in High-Risk Populations, in: Current Concepts in Pediatric Critical Care 2015 . 2015. Eds MR Rigby and AL Graciano.

11. Beghetti M, Schulze-Neick I, Berger RM, et al. Haemodynamic characterization and heart catheterization complications in children with pulmonary hypertension: Insights from the Global TOPP Registry (tracking outcomes and practice in paediatric pulmonary hypertension). Int J Cardiol. 2016 Jan 15;203:325-30

12. Frank BS, Ivy DD. Diagnosis, Evaluation and Treatment of Pulmonary Arterial Hypertension in Children. Children (Basel). 2018 Mar 23;5(4)

13. Hansmann G, Koestenberger M, Alastalo T-P, et al. 2019 updated consensus statement on the diagnosis and treatment of pediatric pulmonary hypertension: The European Pediatric Pulmonary Vascular Disease Network (EPPVDN), endorsed by AEPC, ESPR and ISHLT. J Heart Lung Transplant. 2019 Sep;38(9):879-901.

14. Puri V, Epstein D, Raithel SC, et al. Extracorporeal membrane oxygenation in pediatric lung transplantation. J Thorac Cardiovasc Surg. 2010 Aug;140(2):427-32.

15. Gulack BC, Hirji SA, Hartwig MG. Bridge to lung transplantation and rescue post-transplant: the expanding role of extracorporeal membrane oxygenation J Thorac Dis. 2014 Aug;6(8):1070-9.

16. de Perrot M, Granton JT, McRae K, et al. Outcome of patients with pulmonary arterial hypertension referred for lung transplantation: a 14-year single-center experience. J Thorac Cardiovasc Surg 2012; 143: $910-918$.

17. Huddleston CB, Bloch JB, Sweet SC, et al. Lung Transplantation in Children. Ann Surg. 2002 Sep; 236(3): $270-276$.

18. Williams GD, Ramamoorthy C. Anesthesia considerations for pediatric thoracic solid organ transplant. Anesthesiol Clin N Amer 2005: 23: 709-731.

19. Ius F, Kuehn C, Tudorache I, et al. Lung transplantation on cardiopulmonary support: venoarterial extracorporeal membrane oxygenation outperformed cardiopulmonary bypass. J Thorac Cardiovasc Surg 2012; 144: 1510-1516

20. Moser B, Jaksch P, Taghavi S, Murako zy G, Lang G, Hager H et al. Lung transplantation for idiopathic pulmonary arterial hypertension on intraoperative and postoperatively prolonged extracorporeal membrane oxygenation provides optimally controlled reperfusion and excellent outcome. Eur J 
Cardiothorac Surg 2018; 53:178-185

21. Spray TL, Mallory GB, Canter CE, Huddleston CB, Kaiser LR. Pediatric lung transplantation for pulmonary hypertension and congenital heart disease. Ann Thorac Surg. 1992 Aug;54(2):216-23; discussion 224-5.

22. Bridges ND, Clark BJ, Gaynor JW, Spray TL. Outcome of children with pulmonary hypertension referred for lung or heart lung transplantation. Transplantation. 1996 Dec 27;62(12):1824-8.

23. Mendeloff EN, Meyers BF, Sundt TM, et al. Lung transplantation for pulmonary vascular disease. Ann Thorac Surg. 2002 Jan;73(1):209-17; discussion 217-9.

24. Görler H, Strüber M, Ballmann M, et al. Lung and heart-lung transplantation in children and adolescents: a long-term single-center experience. J Heart Lung Transplant. 2009 Mar;28(3):243-8.

25. Goldstein BS, Sweet SC, Mao J, Huddleston CB, Grady RM. Lung transplantation in children with idiopathic pulmonary arterial hypertension: an 18-year experience. J Heart Lung Transplant. 2011 Oct;30(10):1148-52.

26. Schaellibaum G, Lammers AE, Faro A, Moreno-Galdo A, Parakininkas D, Schecter MG, Solomon M, Boyer D, Conrad C, Frischer T, Wong J, Boehler A, Benden C. Bilateral lung transplantation for pediatric idiopathic pulmonary arterial hypertension: A multi-center experience. Pediatr Pulmonol. 2011 Nov;46(11):1121-7.

27. Hubbard R, Miller R, Tumin D, Tobias JD, Hayes D Jr. Transplant outcomes for idiopathic pulmonary hypertension in children. J Heart Lung Transplant. 2019 May;38(5):580-581.

28. Hayes D Jr, Cherikh WS, Chambers DC, Harhay MO, Khush KK, Lehman RR, Meiser B, Rossano JW, Hsich E, Potena L, Sadavarte A, Singh TP, Zuckermann A, Stehlik J; International Society for Heart and Lung Transplantation. The International Thoracic Organ Transplant Registry of the International Society for Heart and Lung Transplantation: Twenty-second pediatric lung and heart-lung transplantation report-2019; Focus theme: Donor and recipient size match. J Heart Lung Transplant. 2019 Oct;38(10):1015-1027.

29. Schafer M, Wilson N, Ivy DD, Ing R, et al. Noninvasive wave intensity analysis predicts functional worsening in children with pulmonary arterial hypertension. Am J Physiol Heart Circ Physiol. 2018 Oct 1;315(4):H968-H977

\section{Figure Legends}

Figure 1. Survival using the Kaplan-Meier method by diagnosis in pediatric lung transplants perfromed between January 1992 - June 2017. CF = cystic fibrosis, ILD = interstitial lung disease, OB = obliterative bronchiolitis, Retx $=$ retransplant, IPAH $=$ idiopathic pulmonary arterial hypertension, $\mathrm{PH}=$ pulmonary hypertension

Modified from Hayes et $\mathrm{al}^{27}$ with permissionfrom the International Society of Heart and Lung Transplantation.

Figure 2. Survivial using the Kaplan-Meier method conditional on one-year surivival by diagnosis in pediatric lung transplants perfromed between January 1992 - June 2017. CF $=$ cystic fibrosis, ILD = interstitial lung disease, $\mathrm{OB}=$ obliterative bronchiolitis, $\operatorname{Retx}=$ retransplant, $\mathrm{IPAH}=$ idiopathic pulmonary arterial hypertension, $\mathrm{PH}=$ pulmonary hypertension

Modified from Hayes et $\mathrm{al}^{27}$ with permissionfrom the International Society of Heart and Lung Transplantation. 

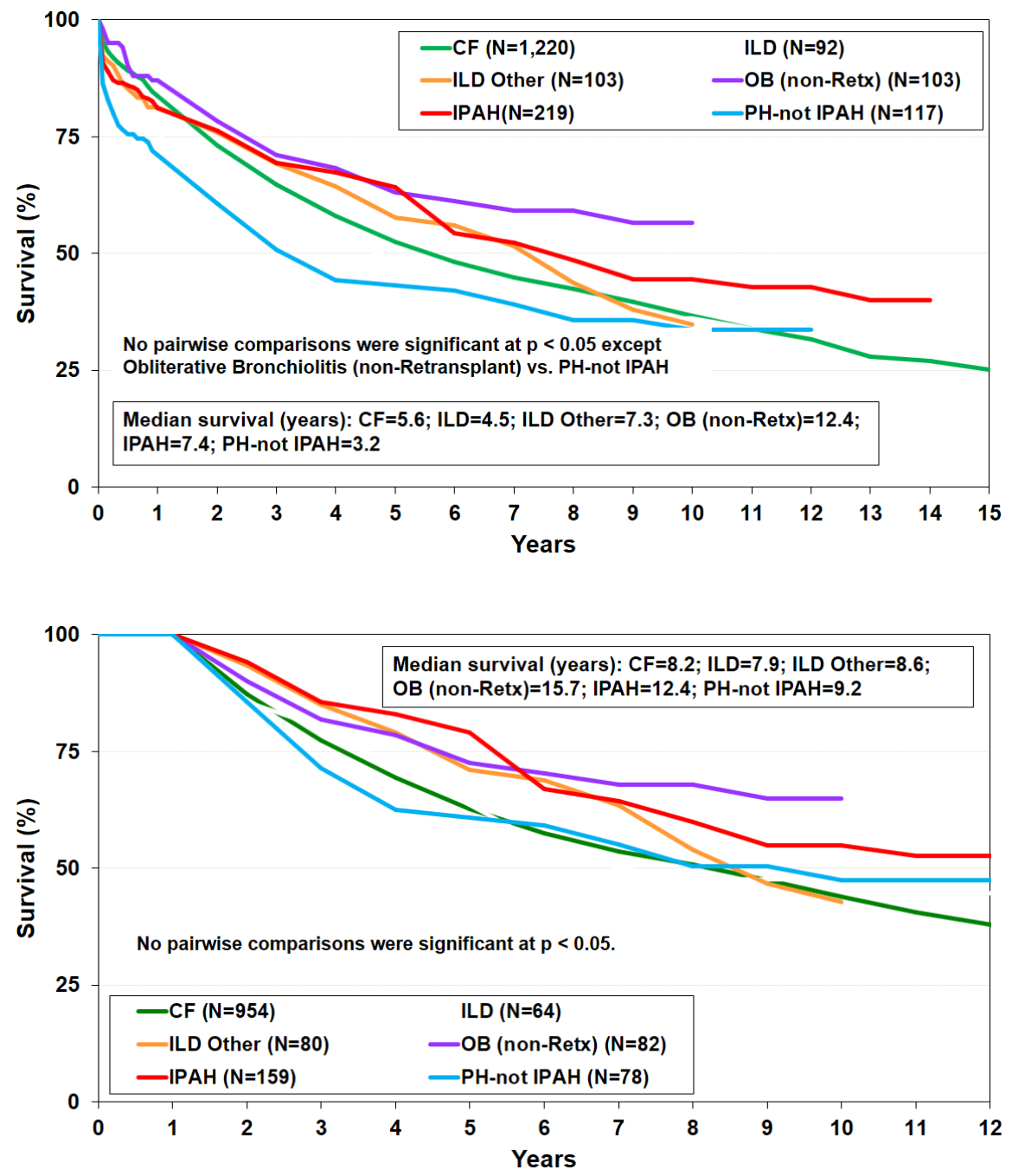